
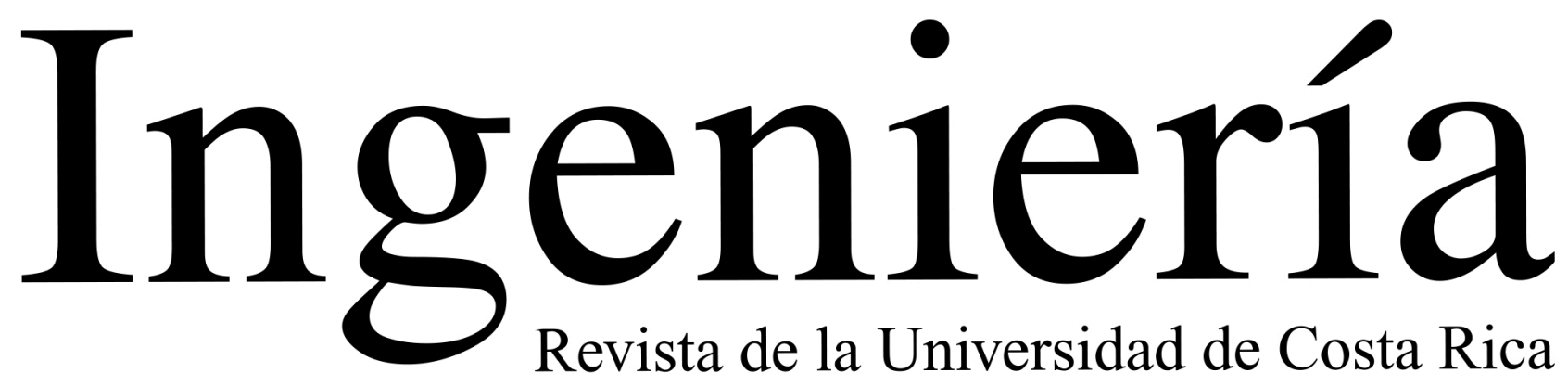

Revista de la Universidad de Costa Rica JULIO/DICIEMBRE 2021 - VOLUMEN 31 (2)

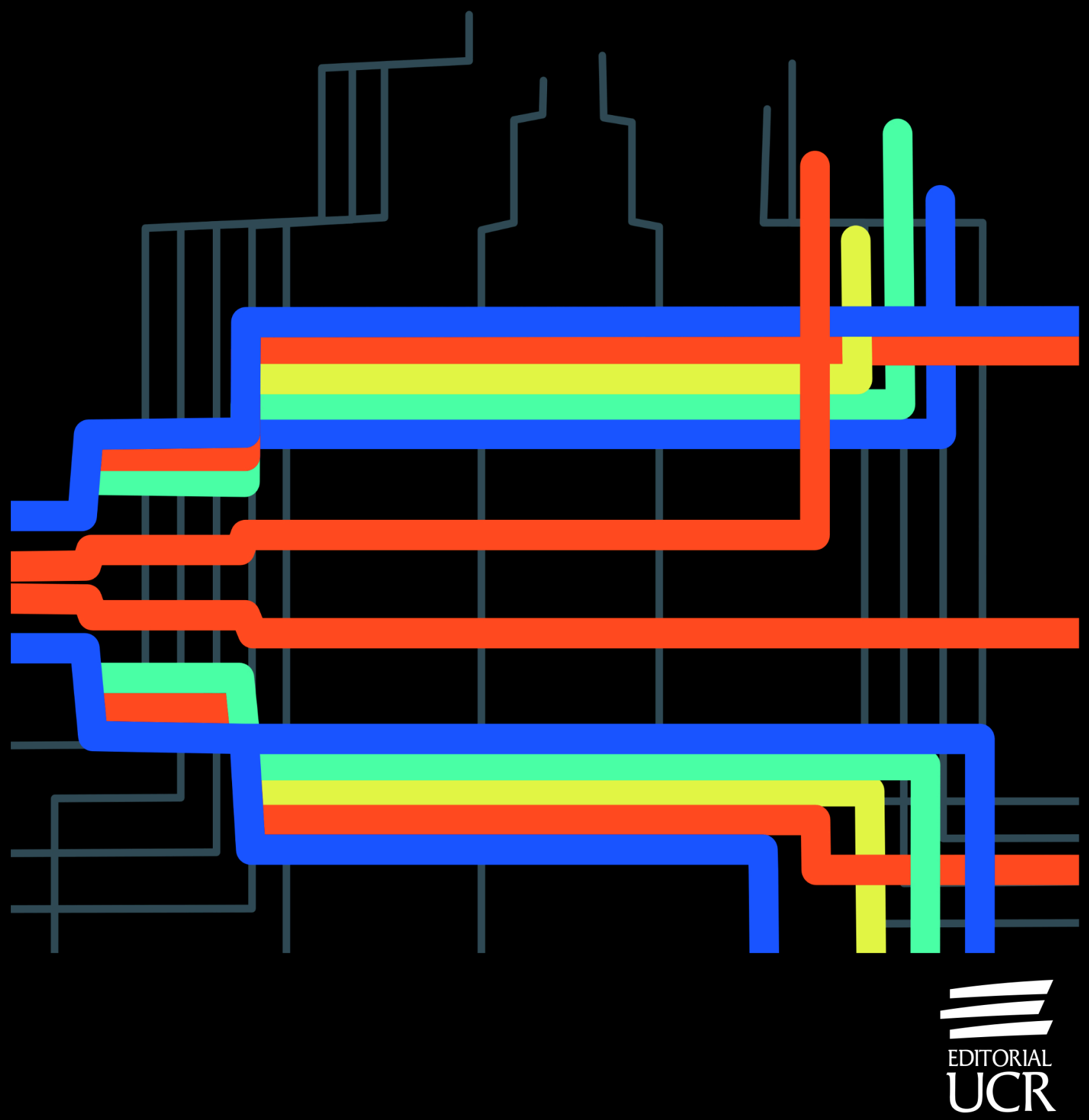




\title{
Evaluación del efecto del pretratamiento del rastrojo de piña para la producción de hidrógeno vía reformado en fase acuosa (APR)
}

\section{Evaluation of the effect of the treatment with diluted sulfuric acid of the lignocellulosic material of pineapple waste to produce sugars as a substrate in the aqueous phase reforming reaction (APR)}

\author{
Jimena Jaikel Viquez \\ Dirección de proyectos en RIVILab, Heredia, Costa Rica \\ Email: jimejaikel@gmail.com \\ ORCID:0000-0002-2608-9205 \\ Adolfo Ulate Brenes \\ Escuela de Ingeniería Química, Universidad de Costa Rica, Costa Rica \\ Email: adolfo.ulate@ucr.ac.cr \\ ORCID:0000-0002-9167-7396
}

Recibido: 18 de agosto 2020

Aceptado: 8 de febrero 2021

\section{Resumen}

La finalidad de este estudio fue evaluar el uso del producto del pretratamiento de material lignocelulósico del rastrojo de piña, para la producción de azúcares, como sustrato en la reacción de reformado en fase acuosa (APR); esto con el fin de producir hidrógeno utilizando un catalizador de níquel soportado en alúmina.

El proceso experimental se realizó en tres etapas: la caracterización del rastrojo de piña utilizado (MD2); hidrólisis del restrojo de piña, en donde se trabajó con un diseño estadística factorial $2^{3}$ para estudiar el efecto de la concentración de ácido sulfúrico diluido $(1 \% \mathrm{~m} / \mathrm{m}$ y $3 \% \mathrm{~m} / \mathrm{m})$, temperatura $\left(100{ }^{\circ} \mathrm{C}\right.$ y $\left.140{ }^{\circ} \mathrm{C}\right)$ y relación sólido-líquido $(1: 6 \mathrm{~g} / \mathrm{mL}$ y $1: 9 \mathrm{~g} / \mathrm{mL})$. Finalmente, la reacción APR que utilizó $5 \%$ de masa de catalizador con respecto a la masa de glucosa, una temperatura de reacción de $473.15 \mathrm{~K}$ y un tiempo de 2 horas. Se varió el porcentaje de níquel en tres niveles: $5 \%, 20 \%$ y $35 \%$.

La mejor condición experimental de la hidrólisis produjo $1.72 \mathrm{~g}_{\text {glucosa }} / 100 \mathrm{~mL}$, utilizando $3 \% \mathrm{~m} / \mathrm{m}$ de ácido sulfúrico diluido, $140{ }^{\circ} \mathrm{C}$ y una relación sólido a líquido de $1 \mathrm{~g}$ a $6 \mathrm{~mL}$. Adicionalmente, se encuentra que la tasa de producción de hidrógeno máxima fue de $221 \mathrm{mmol} \mathrm{g}_{\text {cat }}^{-1} \mathrm{~h}^{-1}$, existiendo diferencia estadística con el catalizador con $35 \%$ de níquel. Además, se realizaron pruebas de la demanda química de oxígeno (DQO), generando valores tanto para el rastrojo hidrolizado de $3032 \mathrm{mg} / \mathrm{L}$, como de la fase líquida de la reacción APR de $466 \mathrm{mg} / \mathrm{L}$. 


\title{
Palabras clave:
}

Biomasa; biocombustibles, catalizador níquel, materiales lignocelulósicos, desplazamiento agua-gas (WGS), aprovechamiento agroindustrial

\begin{abstract}
The main objective of the study was to evaluate the rate of production of hydrogen through aqueous phase reforming (APR), using the product of the hydrolysis of lignocellulosic material of pineapple straw.

There were three phases in this project: the characterization of the pineapple straw (MD2), the hydrolysis of the pineapple straw. It was carried out a fractional factorial design, to study the effect of diluted sulfuric acid concentration $(1 \% \mathrm{~m} / \mathrm{m}$ y $3 \% \mathrm{~m} / \mathrm{m})$, temperature $\left(100{ }^{\circ} \mathrm{C} \mathrm{y} 140{ }^{\circ} \mathrm{C}\right)$ and solid-liquid ratio $(1: 6 \mathrm{~g} / \mathrm{mL}$ y $1: 9 \mathrm{~g} / \mathrm{mL}$ ). The third phase was APR reaction, with $5 \%$ mass of catalyst based on the glucose mass. The temperature $(473.15 \mathrm{~K})$ and the time of reaction $(2 \mathrm{~h})$ were set, and the mass of nickel varied in three levels $(5 \%, 20 \%$ y $35 \%)$.

The highest concentration of glucose in the hydrolysis $(1.72 \mathrm{~g} / 100 \mathrm{~mL}$ of dissolution) were found using $3 \% \mathrm{~m} / \mathrm{m}$ of diluted sulfuric acid, a temperature of $140{ }^{\circ} \mathrm{C}$ and a solid: liquid relation of $1 \mathrm{~g}$ to $6 \mathrm{~mL}$. The rate of hydrogen production was $221 \mathrm{mmol} \mathrm{g}_{\text {cat }}{ }^{-1} \mathrm{~h}^{-1}$, in the APR reaction. According to the statistical design, it was observed that $35 \%$ of nickel is significant. Finally, the chemical oxygen demand (COD) of the hydrolyzed pineapple waste and of the liquid phase of the APR was determined obtaining $3032 \mathrm{mg} / \mathrm{mL}$ and $466 \mathrm{mg} / \mathrm{mL}$, respectively.
\end{abstract}

\section{Keywords:}

Biomass; biofuels, lignocellulosic materials, water gas shift reaction (WGS), nickel catalyst, utilization of agroindustrials residues 


\section{INTRODUCCIÓN}

El petróleo y sus derivados tienen un alto impacto en la economía mundial, además de un papel importante en el medio ambiente y la salud. A pesar de los impactos negativos, el consumo de este no disminuye y sus reservas se reducen rápidamente. [1] El interés en la investigación de la producción de estos recae en la búsqueda de una mayor disponibilidad energética, disminución en la contaminación por emisiones de gases de efecto invernadero, creación de empleos, mecanismos de incentivación de los procesos agroindustriales y manipulación de recursos con mayor conciencia ecológica.

Existe una amplia gama de procesos y materias primas que permite producir combustibles alternos a los derivados del petróleo que, contrario a este, tiende a aumentar su uso. Dentro de la lista se pueden citar residuos orgánicos, cultivos relacionados con azúcares y almidones, material lignocelulósico, plantas oleaginosas y biomasa acuática. Los procesos de transformación de estas biomasas están relacionados con las posibilidades tecnológicas. Un ejemplo es el hidrógeno producido a partir de azúcares provenientes de biomasa que es una alternativa que se está explorando en la actualidad; puesto a que posee como ventajas el hecho de que este brinda seguridad energética, permite una disminución en la contaminación ambiental al ser libre de emisiones y puede utilizarse en motores de combustión interna sin incurrir en altas inversiones monetarias [2], [3].

El proceso de obtención de combustibles alternativos a partir del subproducto de la biomasa ofrece como ventaja una posible estrategia para el aprovechamiento de las grandes cantidades de residuos agroindustriales producidos a nivel mundial. Además, el uso de material lignocelulósico en la fabricación de combustible contribuye a la utilización de biomasas no comestibles para la producción de energía. Estos materiales lignocelulósicos están constituidos por celulosa, hemicelulosa y lignina, los cuales se pueden convertir en azúcares para la producción de energía [4]. La producción de hidrógeno vía biomasa podría, además, satisfacer la demanda en otros mercados como lo son el refinamiento de petróleo, la producción de fertilizantes, producción de metanol, industria metalúrgica y de alimentos [5], [6].

Según lo expuesto por Hernández-Chaverri [7], en Costa Rica se estima que existen 43000 hectáreas de plantación dedicadas al cultivo de piña, lo que representa el séptimo monocultivo de acuerdo con área sembrada; además, convierte a Costa Rica en el primer productor de piña a nivel mundial. Lo anterior genera 642300 toneladas de rastrojo de piña. Aproximadamente, un $65 \%$ de este fruto representa un desecho industrial del mismo, incluyéndose las partes como el corazón, las cáscaras, la corona y hojas [8]. Debido a los altos volúmenes de desechos de la planta, y a la lenta velocidad del proceso de degradación de esta, actualmente se implementan agentes químicos de carácter tóxico como el dicloruro de dimetil-4,4-bipiridilo (Paraquat) para la desecación previa al descarte [9]. Estudios realizados por el Centro de Investigación en Contaminación Ambiental (CICA) de la Universidad de Costa Rica evaluó plaguicidas en aguas superficiales y subterráneas en zonas de cultivo de piña entre los años2015 y 2017. Se encontraron herbicidas como el bromacil, la ametrina y el diurón [10]. Debido a lo anterior, es 
de vital importancia el estudio de métodos alternos y provechosos para los residuos de la piña en búsqueda de la reducción en la contaminación y aumento en las actividades económicas.

\section{ASPECTOS GENERALES}

\subsection{Producción de azúcares a partir del proceso de hidrólisis}

La hidrólisis ácida diluida se ha visto como una opción favorable para el pretratamiento de materiales lignocelulósicos; empleándose usualmente ácido clorhídrico, nítrico, fosfórico y sulfúrico en el proceso [11]. En la mayoría de los casos el pretratamiento con ácido diluido de los materiales lignocelulósicos facilita la liberación de más del $80 \%$ de los azúcares asociados con la fracción de hemicelulosa [12]. El proceso puede efectuarse en dos modalidades: a partir de un proceso a altas temperaturas (superior a $160{ }^{\circ} \mathrm{C}$ ), flujo continuo y baja carga de sólidos $(5 \% \mathrm{~m} / \mathrm{m}-10 \% \mathrm{~m} / \mathrm{m}$ ) o mediante temperaturas bajas (menores a $160^{\circ} \mathrm{C}$ ), en un reactor por lotes con alta carga de sólidos $(10 \% \mathrm{~m} / \mathrm{m}-40 \% \mathrm{~m} / \mathrm{m})[11]$.

\subsection{Reformado en fase acuosa (APR)}

El reformado en fase acuosa (aqueous phase reforming) es un procedimiento que permite la obtención de moléculas simples de hidrógeno y otros productos químicos de valor agregado a partir de materias primas derivadas de la biomasa (con relación de carbono oxígeno 1 a 1 o cercana), en condiciones de reacción a temperaturas alrededor de $500 \mathrm{~K}$ y presiones de (15 - 50) bar; normalmente en presencia de catalizadores metálicos soportados en materiales no conductores [1]. El uso de la técnica del APR presenta una amplia cantidad de ventajas frente a los métodos tradicionales para la producción de moléculas similares, tales como ser aplicable para materias primas solubles en agua, minimizar las reacciones de descomposición indeseables que se encuentran típicamente cuando los carbohidratos se calientan a temperaturas elevadas; y se elimina la necesidad de vaporizar en tal cantidad el agua como el hidrocarbono oxigenado, reduciendo los requisitos de energía para la producción [13].

Pese a las ventajas que presenta el sistema APR, se debe considerar que las condiciones de actividad, selectividad y estabilidad para los sistemas estudiados aún no son las mejores; asimismo, presenta problemas relacionados con la desactivación del catalizador, así como limitaciones asociadas a los fenómenos de transferencia de masa [14]. El APR toma lugar a temperaturas y presiones donde la reacción la reacción de desplazamiento de agua-gas (WGS, water-gas shift reaction) es favorable, haciendo posible generar hidrógeno con bajas cantidades de $\mathrm{CO}$ en un único reactor químico [15].

En términos generales, la ruta o secuencia de reacción de los procesos APR se ve directamente influenciada por condiciones de trabajo como temperaturas, la concentración de la materia prima, la presión en el sistema de reacción y el catalizador. En el caso de la obtención de hidrógeno cómo producto deseado, se busca promover las reacciones que favorecen la escisión del enlace $\mathrm{C}-\mathrm{C}, \mathrm{O}-\mathrm{H}$, $\mathrm{C}-\mathrm{H}$ (reformado de carbohidratos y reacciones de desplazamiento de agua-gas), frente a reacciones 
de rompimiento del enlace de tipo C-O (formación de productos alternos no deseados para la producción de hidrógeno, y consumo de este) (Fischer-Thropsch, metanación) [16].

En primer lugar, la glucosa reacciona en los enlaces de tipo C-C en el sitio metálico del catalizador por medio del proceso de reformado de carbohidratos (2.1), donde se obtiene monóxido de carbono (a partir de la descarbonilación del sustrato) y la consecuente producción de dióxido de carbono e hidrógeno como producto de la reacción de desplazamiento de agua-gas (2.2) [1]. Posteriormente, ocurren procesos de hidrogenación y deshidrogenación, descarboxilación, descarbonilación, hidratación y deshidratación, así como reacciones de cambio de agua-gas; esto al producirse una serie de carbohidratos de menor tamaño, los cuales son capaces de pasar por el proceso de reformado en fase acuosa [17].

Simultáneamente, existen reacciones de competencia como la metanación para la producción de metano (2.3), la hidrogenación directa del dióxido de carbono produciendo metano por la reacción de Fischer-Thropsch (2.4), la reacción de Boudouard (2.5) causante de la aparición de depósitos carbonosos y la escisión de enlaces $\mathrm{C}-\mathrm{O}$, provocando la transformación de los compuestos hacia ácidos orgánicos, aldehídos y cetonas, los cuales no son fácilmente reformables [18]; [13]. Lo anterior denota cómo se ve afectada la selectividad de la producción de hidrógeno a partir de las limitaciones en la transferencia de masa, resultantes por el consumo de hidrógeno en las reacciones alternas. A su vez, cabe destacar el hecho de que los procesos de deshidrogenación de alcoholes en el sitio metálico para su transformación al aldehído correspondiente representan una etapa limitante en la reacción, mientras que la descarbonilación es considerada una reacción relativamente rápida [19].

$$
\begin{aligned}
\mathrm{C}_{6} \mathrm{H}_{12} \mathrm{O}_{6}+6 \mathrm{H}_{2} \mathrm{O} \leftrightarrow 6 \mathrm{CO}_{2} \mathrm{O}+12 \mathrm{H}_{2} & \Delta H>0 \\
\mathrm{CO}+\mathrm{H}_{2} \mathrm{O} \leftrightarrow \mathrm{CO}_{2}+\mathrm{H}_{2} & \Delta H<0 \\
\mathrm{CO}+3 \mathrm{H}_{2} \leftrightarrow \mathrm{CH}_{4}+\mathrm{H}_{2} \mathrm{O} & \Delta H<0 \\
\mathrm{CO}_{2}+4 \mathrm{H}_{2} \leftrightarrow \mathrm{CH}_{4}+2 \mathrm{H}_{2} \mathrm{O} & \Delta H<0 \\
2 \mathrm{CO}_{2} \leftrightarrow \mathrm{CO}_{2}+\mathrm{C} & \Delta H<0
\end{aligned}
$$

Finalmente, es necesario considerar el efecto de la interacción entre el soporte y el precursor para la elección de los componentes del catalizador, en este caso el soporte comúnmente utilizado es la alúmina $\left(\mathrm{Al}_{2} \mathrm{O}_{3}\right)$, al favorecer la selectividad de la reacción hacia la producción de hidrógeno y no contar con sitios ácidos que puedan catalizar reacciones de deshidratación. La alúmina brinda beneficios tales como una alta estabilidad térmica ante los efectos de la sinterización, alta superficie de distribución para el componente metálico y porosidad adecuada para el acceso a la superficie del sitio activo [20].

Por su parte, la elección del componente metálico radica en el favorecimiento de la reacción de desplazamiento de agua-gas por la ruptura de enlaces $\mathrm{C}-\mathrm{C}$ sobre las reacciones de metanación y Fischer-Tropsch, selectividad y estabilidad deseable de la reacción. Se ha descrito que el platino 
y el níquel son los metales que exhiben mejoras en el rendimiento de la reacción de APR, sin embargo, el níquel se presta como una alternativa atractiva frente al platino al poseer un precio en el mercado más accesible que este y al contar con una alta actividad para la reacción de reformado en fase acuosa [4].

\section{EQUIPO Y METODOLOGÍA EXPERIMENTAL}

\subsection{Equipos principales:}

i. Balanza analítica, modelo M124A, ES 1255M;

ii. Capsula de reducción, capacidad de 35 g;

iii. Cromatógrafo de gases con detector de conductividad térmica (TCD), modelo GC-2014, Shimadzu;

iv. Cromatógrafo líquido de alta eficiencia (HPLC) con columna de intercambio iónico, modelo 1260 Infinity, Agilent Technologies;

v. Espectrofotómetro, modelo DR/2010, Hach;

vi. Reactor de alta presión y temperatura, modelo 4561, Parr Instrument Company.

\subsection{Metodología experimental}

\section{Obtención y preparación de la materia prima}

Se trabajó con rastrojo de piña (hojas), variedad MD2 obtenida de la finca orgánica Oro Verde Quintamani, ubicada en el Bajo Plomo de Acosta, San José, Costa Rica (9 45 $^{\prime} 37.1484^{\prime \prime}$, -84 $^{\circ} 1^{\prime} 9^{\prime}$ 28.1928"). Las hojas se lavaron con agua destilada para eliminar posibles trazas de suciedad y se cortaron en trozos de aproximadamente $1 \times 10^{-2} \mathrm{~m}$, se secaron en secadoras para alimento, con el fin de reducir la humedad a menos del $10 \% \mathrm{~m} / \mathrm{m}$. Una vez pasado el proceso de secado se procedió a extraer la fibra mediante una molienda inicial, lo cual permitió reducir aún más el tamaño. Una vez reducido, se tamizó el rastrojo mediante el uso de un molino de alimentos para obtener un tamaño de partícula de $5 \times 10^{-4} \mathrm{~m}(0.5 \mathrm{~mm})$ necesario para la caracterización y $1 \times 10^{-3} \mathrm{~m}(1 \mathrm{~mm})$ para las corridas experimentales.

\section{Caracterización de la materia prima}

\section{Determinación de cenizas}

Para determinar la cantidad de cenizas presente en el rastrojo se utilizó el procedimiento descrito en la norma ASTM D1102-84. 


\section{Determinación del porcentaje de humedad}

El porcentaje de humedad de la muestra se determinó mediante la implementación de una balanza de humedad, Sartorius (modelo MA35M-230N). Para esto, se colocó en el equipo una muestra de $10 \mathrm{~g}$ de rastrojo y se calentó a una temperatura de $100{ }^{\circ} \mathrm{C}$.

\section{Determinación del contenido de lignina}

Se cuantificó la cantidad de lignina presente mediante la norma ASTM 1106 - 96.

\section{Cuantificación de azúcares extraíbles iniciales}

La cuantificación de los azúcares disponibles o azúcares iniciales se realizó mediante una extracción realizada a $2 \mathrm{~g}$ de rastrojo con $20 \mathrm{ml}$ de agua tipo 1 ultra pura, durante 30 minutos. Se procedió a centrifugar la muestra a 2000 XG, se separaron las fases y se extrajeron $2 \mathrm{ml}$. La muestra se trasvasó a un balón de $10 \mathrm{ml}$ y se aforó con agua tipo 1 ultra pura; por último, esta se filtró por medio de una membrana estéril de $0.45 \mu \mathrm{m}$ a un vial para su posterior cuantificación.

\section{Cuantificación de azúcares}

Las muestras reciben el procedimiento descrito en la sección anterior. Se prepara una curva de calibración para el HPLC. Se utilizan patrones con dos mezclas de carbohidratos: fructuosa - glucosa y xilosa - arabinosa. Inicialmente, se pesaron $50 \mathrm{mg}$ en una balanza analítica de cada uno de los azúcares y se colocaron en un balón aforado de $5 \mathrm{ml}$, llevándose a la marca de aforo con agua tipo 1 ultra pura. Seguidamente, se tomaron alícuotas de $1 \mathrm{ml}$ y se colocaron en 5 viales de $2 \mathrm{ml}$ específicos para el uso del cromatógrafo. Se trabajó con un cromatógrafo con columna de intercambio iónico Rezex RCM - Monosaccharide $\mathrm{Ca}^{+2}$, marca Agilent Technologies 1260 Infinity II LC Systems; esta con una fase móvil de agua, con un flujo de $0.4 \mathrm{ml} / \mathrm{min}$. Se estableció como volumen de inyección $3 \mu \mathrm{L}$ y el método implementado requiere una temperatura de columna y del detector de índice de refracción (RID) de $80{ }^{\circ} \mathrm{C}$ y $43^{\circ} \mathrm{C}$, respectivamente.

\section{Hidrólisis ácida}

Para las corridas experimentales se utilizó el reactor Parr de alta presión y temperatura modelo 4561. La hidrólisis ácida se estudió por medio de un diseño factorial fraccionado $2^{3}$ por duplicado, como se muestra en el Tabla I 
TABLA I

NIVELES DE LAS VARIABLES DE DISEÑO ESTABLECIDAS PARA LA HIDRÓLISIS ÁCIDA

\begin{tabular}{ccc}
\hline Variable & Nivel bajo (-) & Nivel alto $(+)$ \\
\hline Concentración de ácido sulfúrico diluido, $C_{a s} /(\% \mathrm{~m} / \mathrm{m})$ & 1 & 3 \\
Temperatura de hidrólisis, $T_{h} /(\mathrm{K})$ & 373.15 & 413.15 \\
Relación sólido a líquido, $r /(\mathrm{g} \mathrm{a} \mathrm{ml})$ & 1 a 9 & 1 a 6 \\
\hline
\end{tabular}

De acuerdo con Azadi, Inderwildi, Farnood y King [21], se considera ácido diluido para uso a nivel de laboratorio, concentraciones de ácido menores a un $10 \% \mathrm{~m} / \mathrm{m}$ de disolución. Asimismo, para la escogencia de los niveles de esta variable se realizó una revisión bibliográfica [22], [23], [24], en donde se tomó como punto de referencia que a porcentajes superiores al $10 \%$ aumenta la posibilidad de reacciones no deseados, y se requiere de mayor cantidad de reactivos para la neutralización de la disoluciones posteriores para su respectivo pretratamiento; por tanto, se decidió utilizar concentraciones de ácido sulfúrico diluido de $1 \% \mathrm{~m} / \mathrm{m}$ y $3 \% \mathrm{~m} / \mathrm{m}$

Los intervalos de temperatura contemplan estudios que demuestran que, a temperaturas inferiores a $373.15 \mathrm{~K}\left(100{ }^{\circ} \mathrm{C}\right)$, los rendimientos de la hidrólisis son bajos, mientras que a temperaturas superiores a $413.75 \mathrm{~K}\left(140{ }^{\circ} \mathrm{C}\right)$, las reacciones secundarias de descomposición empiezan a ser importantes [11], [25], [22].

Para la determinación de la relación rastrojo disolución se realizaron pruebas preliminares para establecer condiciones en donde todo el rastrojo estuviera mojado, que se pudiera agitar de manera eficaz, y evitando la carbonización de este bajo las condiciones de reacción. Cabe señalar que se utilizó como referencia los estudios de [26] y [24].

Para la realización de las corridas se calentó el equipo hasta las temperaturas de trabajo según fuera el caso $(373.15 \mathrm{~K}$ o $413.15 \mathrm{~K})$ y al alcanzarse esta temperatura se dejó reaccionar el sistema durante 40 minutos y bajo agitación constante de $200 \mathrm{rpm}$.

\section{Producción de hidrógeno}

\section{Preparación del catalizador}

Para la preparación del catalizador se utiliza como soporte alúmina (SIGMA-ALDRICH) a los que se le agregó de nitrato de níquel hexahidratado (SIGMA-ALDRICH, $\geq 97.0$ ), para generar el elemento activo sobre el soporte. La cantidad del nitrato de níquel hexahidratado se establece de acuerdo con las condiciones previstas en el diseño experimental (el porcentaje de níquel en el catalizador para valores de 5, 20 y $35 \%$ ). Con el fin de obtener un catalizador homogéneo se utiliza la técnica de impregnación incipiente; se midió la cantidad requerida de nitrato de níquel hexahidratado, disolviéndose lentamente por adición de la mínima cantidad de agua utilizando un 
gotero. Posteriormente, la disolución se transfiere gota a gota a la alúmina previamente tarada en una cápsula de porcelana y se mezcla con un mortero hasta tener una distribución homogénea. El catalizador formado se pasa a una estufa 15 horas a una temperatura de $378.15 \mathrm{~K}$. El catalizador seco se trasladó a un crisol y se calcinó en la mufla durante 3 horas a una temperatura de 723.15 $\mathrm{K}$. El proceso de reducción se realizó antes de cada corrida para la producción de hidrógeno; para esto se introdujo el catalizador en la cápsula de metal, la cual posee un sistema de entrada y salida de gas en la parte superior. El catalizador se redujo por medio de una mezcla de gas de 5\% hidrógeno y $95 \%$ argón a una razón de $250 \mathrm{ml} / \mathrm{min}$. Además, el proceso se efectúa a una temperatura de $723.15 \mathrm{~K}$ por una duración de 4 horas.

\section{Preparación de la muestra para la reacción APR}

Como sustrato para la producción de hidrógeno se utilizó el producto de la hidrólisis ácida. La preparación de la muestra consistió en centrifugar la mezcla a 2000 XG por 10 minutos para separar la parte sólida de la líquida (fase de interés); después, se pasó por un sistema de filtración al vacío.

\section{Reacción de reformado en fase acuosa}

Se definió la cantidad de catalizador como un 5\% de la masa de glucosa en la mezcla de reacción obtenida de la hidrólisis ácida, la cual debe prepararse de acuerdo con lo explicado en el punto anterior. Se establece una temperatura de reacción fija de 473.15 K y un tiempo de reacción de 2 horas, variando el porcentaje de níquel en el catalizador. Se utilizan como niveles en el porcentaje de níquel 5\%, 20\% y 35\% de acuerdo con estudios previos correspondientes. Cada corrida experimental se realiza por duplicado.

A su vez, se utilizó el reactor Parr de alta presión y temperatura modelo 4561. Para cada corrida se dio el proceso de presurización utilizando nitrógeno hasta alcanzar la presión de $1034 \mathrm{kPa}(150$ psi), previo purgado con nitrógeno. La reacción se realiza a una temperatura de $473.15 \mathrm{~K}\left(200{ }^{\circ} \mathrm{C}\right)$ por un tiempo de 2 horas. Finalizada la reacción se extrae una muestra de gas de $4 \mathrm{ml}$ por medio de una jeringa a través del muestreador acoplado para dicho uso.

La producción de hidrógeno se cuantifica por medio de los moles producidos definidos como $\mathrm{mmol}_{\mathrm{H} 2} \mathrm{~g}_{\mathrm{cat}}^{-1} \mathrm{~h}^{-1}$.

\section{Cuantificación del hidrógeno producido}

Para cuantificar la producción de hidrógeno se aplicó vacío por 5 minutos a viales de $2 \mathrm{~mL}$, donde sería inyectada la muestra una vez finalizada la reacción de APR. Se trabajó con un detector de conductividad térmica (TCD) a una temperatura de $503.15 \mathrm{~K}$, implementando nitrógeno como gas de arrastre en el sistema. Como condiciones de operación del cromatógrafo de gases (GC) se tiene un flujo de gas de $4 \mathrm{~mL} / \mathrm{min}$, una temperatura de inyección de $378.15 \mathrm{~K}$, tiempo de 5 min para cada corrida y el uso de una columna de sílice fundida capilar (Carboxen 1010 Plot $30 \mathrm{~m}$ x 0.53 mm) a una temperatura de $323.15 \mathrm{~K}$. Se realizó una inyección de $250 \mu \mathrm{L}$. Se realizó una curva de 
calibración para obtener una relación entre el área obtenida y el porcentaje volumétrico de hidrógeno, mediante la inyección de mezclas de hidrógeno y aire.

\section{Demanda química de oxígeno $(D Q O)$}

Se realizó una prueba para la determinación de la demanda química de oxígeno (DQO), tanto para la fase líquida obtenida de la hidrólisis ácida, como la del producto de la reacción de reformado en fase acuosa (APR). Para esto se tomó una alícuota de $5 \mathrm{ml}$ del producto de APR y $2.5 \mathrm{ml}$ del de hidrólisis. Las alícuotas se trasvasaron a un balón de $50 \mathrm{ml}$ y se llevó a la marca de aforo mediante agua destilada. Se tomó una muestra de $0.2 \mathrm{ml}$ de cada uno y se agregó a un vial para la detección de DQO (disolución de dicromato de potasio, rango de 0 a 15 000). Se colocaron las muestras en un digestor a $423.15 \mathrm{~K}$ por 1.5 horas y una vez transcurrido el tiempo indicado, se dejaron enfriar los viales a temperatura ambiente evitando el contacto con la luz. Finalmente, se calibró el espectrofotómetro con el vial con agua destilada a una longitud de onda de $620 \mathrm{~nm}$ y se colocaron las muestras en este [27].

\section{ANÁLISIS DE RESULTADOS}

\subsection{Caracterización del rastrojo}

Se realizó la caracterización del rastrojo de piña que se utilizó como sustrato para la producción de azúcares. En el Tabla II se presenta los resultados obtenidos.

TABLA II

CARACTERIZACIÓN DEL RASTROJO DE PIÑA MD2

\begin{tabular}{cc}
\hline Análisis & Valor promedio \\
\hline Cenizas $\left(\% \mathrm{~m} / \mathrm{m}_{\text {seca }}\right)$ & $11.62( \pm 0.08)$ \\
Humedad $(\% \mathrm{~m} / \mathrm{m})$ & $83.75( \pm 0.21)$ \\
Lignina $\left(\% \mathrm{~m} / \mathrm{m}_{\text {seca }}\right)$ & $13.79( \pm 0.16)$ \\
& Glucosa: $0.47( \pm 0.04)$ \\
Concentración de azúcares & Fructuosa: $0.75( \pm 0.07)$ \\
extraíbles iniciales $(\mathrm{g} / 100 \mathrm{~mL})$ & Xilosa: $0.016( \pm 0.004)$ \\
\hline
\end{tabular}

La cantidad de cenizas obtenida $(11.62 \% \mathrm{~m} / \mathrm{m})$ para la piña tipo MD2 concuerda con los valores reportados por Córdoba [28] de $12.06 \% \mathrm{~m} / \mathrm{m}$; sin embargo, en estudios realizados con piña variedad champaka se han reportado porcentajes menores $(4.60 \% \mathrm{~m} / \mathrm{m})$ [9], esto se debe a que el porcentaje de cenizas es dependiente de las características de las prácticas de cultivo, cosecha y transporte de la fruta y de los minerales que se encuentran en el suelo adonde se cultiva [29]. 
Dentro de la caracterización de la variedad de piña MD2 se encontró un porcentaje de lignina de $13.79 \% \mathrm{~m} / \mathrm{m}$ en las hojas. Este valor es menor en comparación con otras materias primas o variedades de piña como: caña de azúcar, $24.3 \% \mathrm{~m} / \mathrm{m}$ [30], tallos de maíz, $22.2 \% \mathrm{~m} / \mathrm{m}$ [31], y piña de tipo champaka, $27.72 \% \mathrm{~m} / \mathrm{m}$ [9], lo que facilitaría el acceso a los sacáridos estructurales por medio de la hidrólisis ácida

Para comprar los azúcares que se producían con el pretratamiento propuesto se extrajo con agua ultra pura los elementos solubles que tiene el rastrojo, y a eso se le llamó azúcares extraíbles iniciales; siendo un indicador de los sacáridos no estructurales que contiene la muestra. En cuanto a la caracterización de azúcares extraíbles iniciales se obtuvo un valor cercano al $6 \% \mathrm{~m} / \mathrm{m}_{\text {seca }}$ como se observa en el Tabla II. Este parámetro se utilizará como referencia para el análisis de los resultados obtenidos en la fase de la hidrólisis. No se encontró referencia de otros estudios con rastrojo para realizar la comparación con lo obtenido de azúcares extraíbles determinados en esta investigación. No obstante, en otras publicaciones que utilizan sustratos distintos para las hojas de palma, como el realizado por Martínez y Fernández de Castro, [32], se determina la cantidad de azúcares y reportan valores que inician en un $1,47 \% \mathrm{~m} / \mathrm{m}$ y aumenta conforme pasa el tiempo hasta un $22.05 \% \mathrm{~m} / \mathrm{m}$ después de un mes, mientras que Chico León y Sandoval Rojas [33] obtienen un valor de $1.23 \%$. La comparación con los resultados obtenidos se da como referencia; ya que otros autores que han utilizado rastrojo de piña no han reportado presencia de azúcares solubles, además la norma que se utiliza para la determinación puede variar, así como la naturaleza del sustrato. En el caso de Chico León y Sandoval Rojas [33], el estudio es para cascarilla de arroz y no detallan la norma que se utiliza. Con base en la información anterior se podría inferir que la presencia de azúcares extraíbles iniciales se relaciona al tiempo y condiciones de almacenamiento de la materia prima.

\subsection{Pretratamiento químico con ácido sulfúrico diluido}

En esta etapa experimental se trabajó con un diseño estadística factorial $2^{3}$ por duplicado, para estudiar el efecto de la concentración de ácido sulfúrico diluido $(1 \% \mathrm{~m} / \mathrm{m}$ y 3\% $\mathrm{m} / \mathrm{m})$, de la temperatura $\left(100{ }^{\circ} \mathrm{C}\right.$ y $\left.140{ }^{\circ} \mathrm{C}\right)$ y de la relación sólido-líquido $(1: 6 \mathrm{~g} / \mathrm{mL}$ y $1: 9 \mathrm{~g} / \mathrm{mL})$ en la hidrólisis ácida en la obtención de azúcares. El tiempo de reacción que se estableció fue de 40 minutos, puesto que, de acuerdo con estudios anteriores, a tiempos mayores, la glucosa comienza un proceso de transformación a otros productos secundarios y en contraste, a tiempos menores no han ocurrido cambios significativos en la materia prima, por lo que aún no se tiene una producción considerable de azúcar [34] y [35].

En la Tabla III se presenta el resultado promedio de concentración de azúcares totales para cada una de las distintas combinaciones del diseño estadístico.

La mejor condición experimental encontrada fue para la temperatura de $140{ }^{\circ} \mathrm{C}$, relación sólido/líquido de 1:6 g/mL, y una concentración de ácido sulfúrico de $3 \% \mathrm{~m} / \mathrm{m}$. En la Figura 1 se muestran los resultados de promedio de concentración de sacáridos para cada una de las distintas combinaciones del diseño estadístico. 


\begin{tabular}{|c|c|c|c|c|c|}
\hline \multicolumn{6}{|c|}{$\begin{array}{c}\text { TABLA III } \\
\text { PROMEDIO DE CONCENTRACIÓN DE AZÚCARES TOTALES OBTENIDO } \\
\text { PARA EL DISEÑO ESTADÍSTICO }\end{array}$} \\
\hline Muestra & $\begin{array}{c}\text { Temperatura } \\
\left({ }^{\circ} \mathbf{C}\right)\end{array}$ & $\begin{array}{l}\text { Relación } \\
\text { sólido/líquido } \\
\quad(g / m l)\end{array}$ & $\begin{array}{c}\text { Concentración } \\
\text { de ácido }(\% \mathrm{~m} / \mathrm{m})\end{array}$ & $\begin{array}{c}\text { Promedio } \\
\text { concentración } \\
\text { de azúcar } \\
\text { total, } \\
\mathrm{C}_{\mathrm{glu}} /(\mathrm{g} / \mathbf{1 0 0} \mathrm{ml})\end{array}$ & $\begin{array}{l}\text { Relación masa } \\
\text { glucosa masa } \\
\text { rastrojo (g } \\
\text { azúcar total/g } \\
\text { rastrojo) }\end{array}$ \\
\hline 1 & 100 & 1 a 6 & 1 & $1.90( \pm 0.08)$ & 0.11 \\
\hline 2 & 100 & 1 a 6 & 3 & $3.19( \pm 0.29)$ & 0.19 \\
\hline 3 & 100 & 1 a 9 & 1 & $1.32( \pm 0.16)$ & 0.12 \\
\hline 4 & 100 & 1 a 9 & 3 & $2.16( \pm 0.18)$ & 0.19 \\
\hline 5 & 140 & 1 a 6 & 1 & $1.85( \pm 0.04)$ & 0.11 \\
\hline 6 & 140 & 1 a 6 & 3 & $3.08( \pm 0.32)$ & 0.18 \\
\hline 7 & 140 & 1 a 9 & 1 & $1.64( \pm 0.32)$ & 0.15 \\
\hline 8 & 140 & 1 a 9 & 3 & $1.99( \pm 0.85)$ & 0.18 \\
\hline $\begin{array}{l}\text { Comprobación } \\
\text { (muestra 6) }\end{array}$ & 140 & 1 a 6 & 3 & $3.06( \pm 0.31)$ & \\
\hline
\end{tabular}

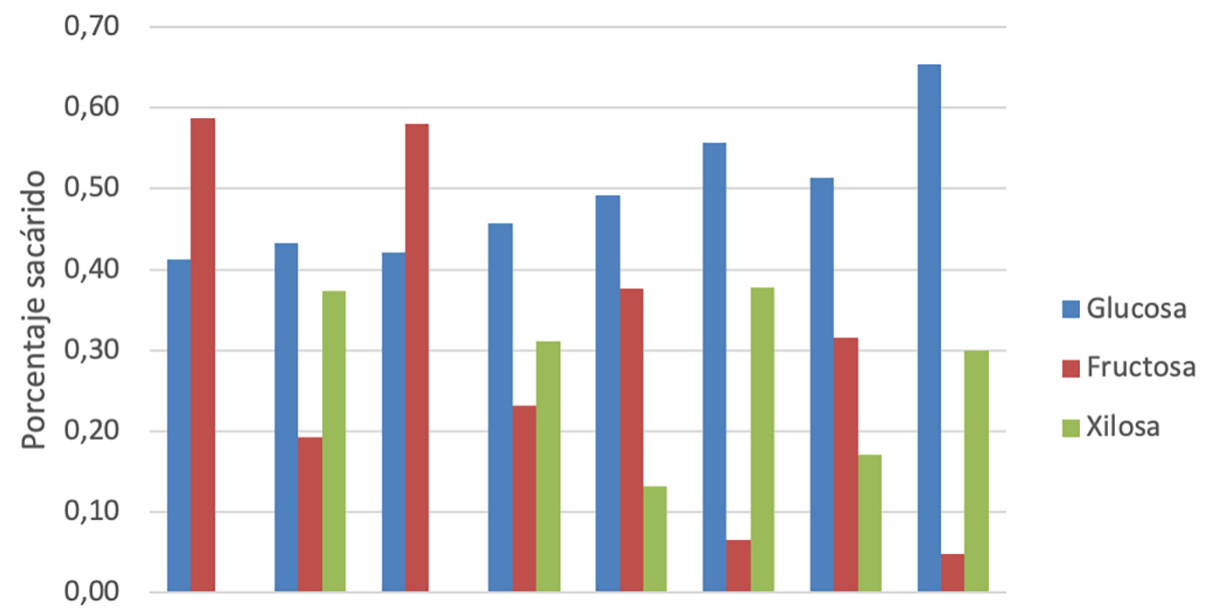

Fig. 1. Distribución de sacáridos obtenidos en la hidrólisis ácida del rastrojo de piña 
El pretratamiento con ácido sulfúrico diluido del material lignocelulósico permite tener mejoras en el rendimiento de producción de glucosa, puesto a que este incrementa el área superficial y el volumen del poro, altera la estructura lignocelulósica al degradar la lignina, solubilizar la hemicelulosa y fragmentar la estructura cristalina de la celulosa [36]. La concentración de glucosa obtenida en la corrida 6 de $1.72 \mathrm{~g}_{\mathrm{glu}} / 100 \mathrm{~mL}_{\text {dis }}\left(10.29 \mathrm{~g}_{\mathrm{glu}} / 100 \mathrm{~g}_{\mathrm{MS}}\right)$ presentó valores superiores a los analizados en estudios realizados con otras materias primas bajo condiciones similares de hidrólisis, como lo son: el aserrín, $3.15 \mathrm{~g}_{\mathrm{glu}} / 100 \mathrm{~g}_{\mathrm{MS}}$ [37] la caña de azúcar, $1.46 \mathrm{~g}_{\mathrm{glu}} / 100 \mathrm{~mL}_{\mathrm{dis}}$ [25], bagazo de arroz, $3.3 \mathrm{~g}_{\mathrm{glu}} / 100 \mathrm{~g}_{\mathrm{MS}}$ [38], pasto, $2.83 \mathrm{~g}_{\mathrm{glu}} / 100 \mathrm{~g}_{\mathrm{MS}}$ [39], moringa, $8.40 \mathrm{~g}_{\mathrm{glu}} / 100 \mathrm{~g}_{\mathrm{MS}}$ [40]. Jung Y. H., Kim I. J., Kim H. K., Kim K. H. [41] para la hidrólisis ácida de racimos de frutos de palma africana (RFPA) obtiene resultados que varían desde $5.5 \mathrm{~g} / 100 \mathrm{~g}$ de RFPA hasta casi $16 \mathrm{~g} / 100 \mathrm{~g}$ de RFPA. Por su parte, Choojit S., Ruengpeerrakul T., Sangwichien C [42], por medio de un pretratamiento ácido a hojas de piña en condiciones similares obtienen $15.37 \mathrm{~g} / \mathrm{L}$ de glucosa, estando dentro del intervalo obtenido en este estudio.

La Tabla IV presenta los resultados del análisis estadístico utilizando un nivel de confianza del $95 \%$. Se encontró que la variable significativa en la producción de azúcares totales es la concentración de ácido sulfúrico diluido, que provoca un aumento del 67 \% para la producción de azúcares totales producida cuando se aumenta la concentración del nivel menor al mayor.

TABLA IV

CÁLCULOS ESTADÍSTICOS PARA EL DISEÑO FACTORIAL $2^{3}$

\begin{tabular}{ccccc}
\hline Fuente & Efecto & $\begin{array}{c}\text { Cuadros medios ajusta- } \\
\text { dos }\end{array}$ & Factor F & Probabilidad \\
\hline A & 0.01661265 & 0.01661265 & 16.9793412 & 0.003341441 \\
B & 0.00039031 & 0.00039031 & 0.39892936 & 0.545263379 \\
$\mathrm{C}$ & $3.7 \times 10^{-6}$ & $3.7 \times 10^{-6}$ & 0.48049021 & 0.952413153 \\
$\mathrm{AB}$ & 0.00047011 & 0.00047011 & 0.00379127 & 0.507821341 \\
& & & & \\
$\mathrm{AC}$ & 0.00057771 & 0.00057771 & 0.59046266 & 0.464317071 \\
$\mathrm{BC}$ & 0.00013374 & 0.00013374 & 0.13669583 & 0.721183763 \\
$\mathrm{ABC}$ & 0.0043911 & 0.0043911 & 0.44879835 & 0.521769490 \\
\hline
\end{tabular}

$\mathrm{A}=$ Concentración de ácido sulfúrico diluido, $\mathrm{Cas} /(\% \mathrm{~m} / \mathrm{m}), \mathrm{Th} /\left({ }^{\circ} \mathrm{C}\right), \mathrm{B}=$ Relación sólido a líquido, $\mathrm{r} /(\mathrm{g} / \mathrm{mL})$, $\mathrm{C}=$ Temperatura de hidrólisis.

Las muestras producidas a partir de la digestión con ácido sulfúrico al $3 \% \mathrm{~m} / \mathrm{m}$ presentaron concentraciones de azúcares totales superiores a aquellas tratadas con ácido diluido al $1 \% \mathrm{~m} / \mathrm{m}$. Esto 
debido a que una mayor concentración de ácido diluido permite solubilizar mejor la hemicelulosa y efectuar el proceso de deslignificación; se considera ácido diluido para concentraciones menores a $10 \% \mathrm{~m} / \mathrm{m}$ de ácido en disolución [21]. La concentración de ácido influye directamente en el pH de la disolución, donde a menor $\mathrm{pH}$ se tiene mayor efecto de protonación en el ataque al enlace $\beta$ $(1 \rightarrow 4)$ glicosídico (el cual une las unidades de monómero de $\beta$-anhidroglucosa entre sí), permitiendo debilitar el enlace para facilitar la separación de la molécula en azúcares simples. Lo anterior, a fin de aumentar el acceso a la red cristalina de la celulosa presente y permitir el rompimiento de los enlaces carbono-oxígeno para la conversión del sustrato en los azúcares objetivo [11].

En el caso del efecto de la temperatura, la concentración de azúcares totales producida tiende a incrementar a medida que aumenta esta variable; esto, pues, las mayores temperaturas permiten acelerar la velocidad de hidrólisis y consecuentemente la tasa de formación de la azúcares. Además, esta permite catalizar el efecto del ácido en términos de la degradación de la lignina, despolimerización de la hemicelulosa y digestión de la estructura de la celulosa (concordando con la corrida experimental con mayor producción de azúcares) [43]. Sin embargo, de acuerdo con los resultados del diseño estadístico no se presentaron diferencias significativas para la temperatura, lo cual se estima que podría atribuirse al hecho de que a condiciones de estudio se dan en un intervalo de variación pequeño, condiciones seleccionadas debido a que a temperaturas mayores, las pentosas y hexosas producidas comienzan a transformarse en productos secundarios no deseados [23].

Respecto al efecto de la relación de sólidos y licor de reacción en el sistema, no existen diferencias significativas en términos de los dos niveles escogidos para la variable. Lo que se evidencia porque el cambio del nivel menor al mayor representa una variación de $0,01 \mathrm{~g}$ de glucosa por cada gramo de rastrojo. Basado en estos resultados, se considera adecuado trabajar con una carga de sólidos superior (relación $1 \mathrm{~g}$ de rastrojo con $6 \mathrm{ml}$ de disolución), para la siguiente etapa experimental ya que cumple con las condiciones mínimas de cantidad de azúcares totales para la reacción de APR; adicionalmente, es un proceso con menor impacto ambiental ya que se utiliza menor cantidad de disolución lo que significa a una disminución en el consumo posterior de reactivos para la neutralización y tratamiento de desechos.

En términos de las interacciones entre las variables estudiadas, de acuerdo con la Tabla IV, son no significativas. Sin embargo, los resultados de la interacción sólido-líquido y temperatura (BC) en los niveles mayores del estudio, y una vez terminado el experimento, presentaron una consistencia más líquida. Lo anterior favorece el mezclado de los compuestos y una disminución en la viscosidad, algo favorable para el proceso de transferencia de masa en la reacción, lo que concuerda con lo encontrado con lo encontrado por Chen [44]. Además, la relación entre la variable temperatura y la variable relación de sólidos se estudió en pruebas preliminares, en donde quedó en evidencia que con mayor cantidad de sustrato, la disponibilidad de humedad disminuye, y al aumentar la temperaturas, el rastrojo es susceptible a experimentar carbonización. 


\subsection{Reacción de reformado en fase acuosa (APR)}

El objetivo de esta etapa experimental reside en evaluar el efecto en la actividad catalítica de la variación en el porcentaje de níquel impregnado en un soporte de alúmina, para la producción de hidrógeno mediante una reacción de reformado en fase acuosa. Con los resultados obtenidos en la fase anterior de hidrólisis, se utilizó la mezcla de reacción producto de la corrida experimental con mayor porcentaje de glucosa como reactivo de partida para el proceso APR.

Todas las corridas experimentales de esta etapa utilizaron como base la mezcla del proceso anterior con un porcentaje de $1.62 \% \mathrm{~m} / \mathrm{m}$ de glucosa, el cual es equivalente a un $2.88 \% \mathrm{~m} / \mathrm{m}$ de la mezcla de azúcares totales. De acuerdo con la literatura, es posible la producción de hidrógeno por medio de la reacción de APR de subproductos de hidrólisis ácida; tales como, fructuosa, ácido acético, glucosa, xilosa y furfural [14]. Los valores utilizados en esta etapa se ajustan al intervalo propuesto por Cortright [17] de $1 \% \mathrm{~m} / \mathrm{m}$ y $10 \% \mathrm{~m} / \mathrm{m}$. El intervalo anterior utiliza concentraciones bajas, puesto a los azúcares pueden experimentan reacciones secundarias en la fase líquida, formando compuestos como aldehidos, ácidos orgánicos y depósitos de carbono.

De acuerdo con Tanksale [45], las condiciones recomendadas para la producción de hidrógeno a partir de biomasa en un sistema de reacción por lotes se encuentran a temperaturas entre los 185 ${ }^{\circ} \mathrm{C}$ y $220^{\circ} \mathrm{C}$, con presiones que varían entre los 25 bar y 40 bar. A temperaturas mayores a $220{ }^{\circ} \mathrm{C}$, la glucosa se vuelve inestable y comienza un proceso de desactivación después de 3 horas debido a la formación de productos intermediarios (éteres aromáticos, grupos ésteres), los cuales no toman parte en la reacción de APR; así como, ocurre la formación de restos de coque en el sistema (ocasiona bloqueos en el área superficial del metal). Temperaturas menores promueven la producción de alcoholes y ácidos ( $\mathrm{C} 1$ a C4) por la división de los enlaces C-O presentes [46].

La Tabla V muestra el resultado de la generación de hidrógeno, donde se observa una relación entre la producción de hidrógeno y la cantidad de níquel en el catalizador.

TABLA V

RESULTADOS OBTENIDOS PARA LA PRODUCCIÓN DE HIDRÓGENO EN LA REACCIÓN APR

\begin{tabular}{cc}
\hline $\begin{array}{c}\text { Porcentaje de metal en } \\
\text { el catalizador } /(\% \mathbf{m} / \mathbf{m})\end{array}$ & $\begin{array}{c}\text { Generación de hidrógeno, } \boldsymbol{G} / \\
\left(\mathbf{m m o l} / \mathbf{g}_{\text {cat }} \mathbf{h}\right)\end{array}$ \\
\hline 5 & 110 \\
20 & 149 \\
35 & 221 \\
\hline
\end{tabular}

Se encontraron diferencias estadísticamente significativas en la tasa de generación de hidrógeno según los niveles escogidos para la variación del porcentaje de metal en el catalizador $(5 \% \mathrm{~m} / \mathrm{m}$, 
$20 \% \mathrm{~m} / \mathrm{m}$ y $35 \% \mathrm{~m} / \mathrm{m})(\mathrm{F}=14.71 ; \mathrm{gl}=2 ; \mathrm{p}=0.001)$. Seguidamente, se realizó la prueba de Tukey de comparación múltiple, la cual agrupó a los porcentajes de metal en dos grupos: (1) 5 y $20 \%$ m/m y (2) $35 \% \mathrm{~m} / \mathrm{m}$.

De acuerdo con el análisis estadístico, se recomienda trabajar con un porcentaje de níquel en el catalizador de $35 \% \mathrm{~m} / \mathrm{m}$, ya que este exhibió un mejor desempeño en términos de la tasa de producción del hidrógeno. Esto puede atribuirse a un incremento en la actividad catalítica, resultante de una mayor cantidad de sitios activos disponibles, los cuáles favorecen la reacción de reformado en fase acuosa y el aumento en la selectividad a hidrógeno [16]. La cantidad considerable de sitios activos se relaciona con una mejor distribución del metal en la superficie catalítica, así como, una mayor porosidad y área superficial alta [47].

Los resultados encontrados concuerdan con el estudio de Wen et al. [48], donde se probaron catalizadores de $\mathrm{Ni} / \mathrm{Al}_{2} \mathrm{O}_{3}$ variando la carga de níquel desde un $14 \% \mathrm{~m} / \mathrm{m}$ a un $48 \% \mathrm{~m} / \mathrm{m}$ y se encontró que las muestras con mayor cantidad metálica $(36 \% \mathrm{~m} / \mathrm{m}$ y $48 \% \mathrm{~m} / \mathrm{m})$ poseían altas actividades catalíticas para la reacción APR de la glucosa. En el estudio se presenta una producción de $46.9 \%$ mol de $\mathrm{H}_{2}$ con una selectividad de hidrógeno de $43.5 \%$ para la carga de $48 \% \mathrm{~m} / \mathrm{m}$ y $41.7 \%$ mol de $\mathrm{H}_{2}$ con una selectividad hacia el hidrógeno de $35.2 \%$ para el porcentaje de 36 $\% \mathrm{~m} / \mathrm{m}$. Se obtiene, que la generación de hidrógeno producida tiene un valor promedio de 259 mmol h $\mathrm{h}_{\text {cat }}^{-1}$ para el $35 \%$ de níquel en el catalizador, y es similar a la reportada por los estudios que utilizan materias primas similares como el Pipitone et al. [49] para una mezcla de glucosa y xilosa, $42.67 \mathrm{mmol} \mathrm{h}^{-1} \mathrm{~g}_{\text {cat }}{ }^{-1}$; y superiores a otros estudios como el de Coronado et al. [47] para el metanol de $0.516 \mu \mathrm{mol} \mathrm{h}^{-1} \mathrm{~g}_{\text {cat }}{ }^{-1}$, Wen et al. [50] para glicerol $10.06 \mathrm{mmol} \mathrm{h}^{-1} \mathrm{~g}_{\text {cat }}{ }^{-1} \mathrm{y}$ Meryemoglu et al. [51] para paja de trigo, $40 \mathrm{mmol} \mathrm{h}^{-1} \mathrm{~g}_{\text {cat }}{ }^{-1}$. Sin embargo, este estudio está utilizando un sustrato de reacción del pretratamiento ácido, en donde no solo se producen los azúcares, si no, que hay remanentes de reacciones secundarias del proceso como se mencionó antes, a diferencia de sustratos sintéticos en donde solo se estudia el caso del compuesto de interés (como los que se comparan). Otro aspecto que se debe de anotar es la gran cantidad de residuos sólidos que se forman en el sistema de reacción que cubren al catalizador, por lo que su capacidad de trabajar disminuye. Esta condición lo presentan otros como se menciona anteriormente.

\subsection{Demanda química de oxígeno (DQO)}

Se determinó el DQO para la fase líquida producto de la hidrólisis ácida y de reformado en fase acuosa, con el fin de establecer en un primer intento, el impacto ambiental que pueda presentar el proceso de producción de hidrógeno. En la Tabla VI se observa como la muestra resultante de la hidrólisis posee un valor 6.5 veces mayor a la muestra después del proceso de reformado en fase acuoso. Los valores producto de la hidrólisis están de acuerdo con lo esperado, ya que se están liberando azúcares, ácidos orgánicos y otro tipo de moléculas producto de la degradación. Esto además concuerda con la complejidad de la composición la mezcla producto de la hidrólisis. 
TABLA VI

DQO DE LAS MUESTRAS ANALIZADAS

\begin{tabular}{cc}
\hline Muestra & $\begin{array}{c}\text { Promedio demanda Química } \\
\text { de Oxígeno, } \boldsymbol{D Q O} /(\mathbf{m g} / \mathbf{L})\end{array}$ \\
\hline Producto del rastrojo hidrolizado & 3032 \\
Producto de la reacción de APR & 466 \\
\hline
\end{tabular}

Para el producto líquido de la reacción APR se tiene una disminución del contenido orgánico (expresado como demanda bioquímica de oxígeno) lo que evidencia que la reacción de reformado además de hidrógeno promueve la producción de otros productos gaseosos como dióxido de carbono y metano como se observa en las reacciones (2.1) a (2.5), adicionalmente, junto con el licor de reacción se obtiene un sustrato sólido que posee potencial en otros procesos de aprovechamiento de biomasa. Junto con la determinación del DQO se estableció que durante la reacción de reformado en fase acuosa se dio un consumo de glucosa de un $99.9 \%$, al pasar de una concentración inicial de $1.718 \mathrm{~g} / 100 \mathrm{ml}$ a una final de $0.0017 \mathrm{~g} / 100 \mathrm{ml}$ durante el proceso.

\section{CONCLUSIONES}

- El rastrojo de piña utilizado presenta entre su composición un $11.62 \% \mathrm{~m} / \mathrm{m}$ de cenizas, 13.79 $\% \mathrm{~m} / \mathrm{m}$ de lignina, $83.75 \% \mathrm{~m} / \mathrm{m}$ de humedad y una concentración de azúcares extraíbles iniciales de $0.47 \mathrm{~g} / 100 \mathrm{ml}$ de glucosa, $0.75 \mathrm{~g} / 100 \mathrm{ml}$ de fructuosa y $0.016 \mathrm{~g} / 100 \mathrm{ml}$ de xilosa.

- Para el pretratamiento que se realizó al rastrojo, la única variable significativa desde el punto de vista estadístico fue la concentración del ácido diluido, en donde la mayor concentración (3\%) se obtiene mayor producción de glucosa., bajo las condiciones de una concentración de $3 \% \mathrm{~m} / \mathrm{m}$ de ácido sulfúrico diluido, una temperatura de $140{ }^{\circ} \mathrm{C}$ y una relación sólido a líquido de $1 \mathrm{~g}$ a $6 \mathrm{ml}$, para una concentración de este azúcar de $1.72 \mathrm{~g} / 100 \mathrm{ml}$ de disolución.

- Se logró la producción de hidrógeno a partir del rastrojo de piña, utilizando catalizador de níquel soportado en alúmina. El catalizador con mayor porcentaje de níquel $(35 \% \mathrm{~m} / \mathrm{m})$ exhibió un mejor desempeño en términos de la generación de hidrógeno, con un valor promedio de $259 \mathrm{mmol} / \mathrm{g}_{\text {cat }}{ }^{-1} \mathrm{~h}^{-1}$.

\section{AGRADECIMIENTOS}

A la Red de Investigación y Desarrollo en Eficiencia Energética y Energías Renovables de Universidad de Costa Rica por el apoyo con el préstamo de equipos necesarios para el desarrollo del estudio. 


\section{BIBLIOGRAFÍA}

[1] Y. Wei, H. Lei, Y. Liu, L. Wang, L. Zhu, X. Zhang, G. Yadavalli, B. Ahring y S. Chen, "Renewable Hydrogen Produced from Different Renewable Feedstock by Aqueous-Phase Reforming Process", Journal of Sustainable Bioenergy Systems, vol. 4, pp. 113-127, 2014.

[2] S. Sing , S. Jain, A. Tiwari, M. Nouni, J. Pandey y S. Goel, "Hydrogen: a Sustainable Fuel for Future of the Transport Sector.", Renewwable and Sustainable Energy Reviews, vol. 51, pp. 623-633, 2015.

[3] S. J. Gerssen-Gondelach, D. Saygin, B. Wicke, M. K. Patel y A. Faaij, "Competing uses of biomass: Assessment and comparison of theperformance of bio-based heat, power, fuels and materials", $R e$ newable and Sustainable Energy Reviews, vol. 40, pp. 964-988, 2014.

[4] N. D'Angelo, Aqueous Phase Reforming of Bio-Carbohydrates: reactor engineering and catalysis, Technische Universiteit Eindhoven, 2014.

[5] A. Bakenne, W. Nutall y N. Kazantzis, "Sankey-Diagram-based insights into hydrogen economy today", International Journal of Hydrogen Energy, vol. 41, pp. 7744-7753, 2016.

[6] The Royal Society, Options for producing low-carbon hydrogen at scale, London, 2018.

[7] R. Hernández-Chaverri y L. A. Prado Barragán, "Impacto y oportunidades de biorrefinería de los desechos agrícolas del cultivo de piña (Ananas comosus) en Costa Rica", UNED Research Journal, vol. 10, $\mathrm{n}^{\mathrm{o}} 2$, pp. 455-468, 2018.

[8] M. A. Maglianesi, "Desarrollo de las Piñeras en Costa Rica y sus Impactos Sobre Ecosistemas Naturales y Agro-urbanos”, Biocenosis, vol. 27, pp. 1-2, 2013.

[9] K. Quesada, P. Alvarado, R. Sibaja y J. Vega, "Utilización de las Fibras del Rastrojo de Piña (Ananas comusus, variedad champaka) como Material de Refuerzo en Resinas de Poliéster.", Revista Iberoamericana de Polímeros, vol. 6, nº 2, 2005.

[10] Universidad de Costa Rica, «https://www.ucr.ac.cr/noticias/2018/06/14/ucr-detecta-residuos-deplaguicidas-en-fuentes-de-agua-en-la-zona-norte.html,» UCR, 1406 2018. [En línea]. [Último acceso: 1212 2020].

[11] P. Bajpai, Pretreatment of Lignocellulosic Biomass for Biofuel Production, Singapore: Springer, 2016.

[12] J. R. Mielenz, K. Klasson, W. S. Adney y J. D. McMillan, Biotechnology for Fuels and Chemicals: The Twenty-Eighth Symposium, Estados Unidos: Springer Science and Business Media, 2007.

[13] G. Chen, W. Li, H. Chen y B. Yan, "Progress in the aqueous-phase reforming of different biomass-derived alcohols for hydrogen production," Journal of Zhejiang University-SCIENCE A, vol. 16, pp. 491-506, 2015.

[14] I. Coronado, M. Stekrova, M. Reinikainen, P. Simell y L. Lefferts, "A review of catalytic aqueous-phase reforming of oxygenated hydrocarbons derived from biorefinery water fractions," Hydrogen Energy, pp. 11003-11032, 2016.

[15] R. R. Davda, J. W. Shabaker, R. D. Huber, R. D. Cortright y J. A. Dumesic, "A review of catalytic issues and process conditions for renewable hydrogen and alkanes by aqueos-phase reforming of oxygenated hidrocarbons over supporter metal catalysts," Applied Catalysis B, pp. 171-186, 2005. 
[16] H. A. Duarte, M. E. Sad y C. R. Apesteguía, "Aqueous Phase Reforming of Sorbitol on Pt/A12O3: Effect of Metal Loading and Reaction Conditions on H2 Productivity", International Journal of Hydrogen Energy, vol. 41, nº 39, pp. 17290-17296, 2016.

[17] R. D. Cortright, R. R. Davda y J. A. Dumesic, "Hydrogen from Catalytic Reforming of Biomass-derived Hydrocarbons in Liquid Water", Nature Publishing Group, vol. 418, pp. 964-967, 2002.

[18] G. E. Díez, Producción de Bio-hidrógeno Mediante Gasificación Catalítica de Biomasa con Captura Integrada de CO2 (Tesis para optar por el grado de Doctorado en Ingeniería Energética), Oviedo, España: Universidad de Oviedo, 2007.

[19] A. Kirilin, Aqueous-phase Reforming of Renewables for Selective Hydrogen Production in the Presence of Supported Platinum Catalysts. Laboratory of Industrial Chemistry and Reaction Engineering, Finland: Åbo Akademi University, 2013.

[20] L. Dosso, Sistemas Catalíticos de Pt-Ni-Co para Producción de Hidrógeno por Reformado en Fase Acuosa de Productos de la Hidrólisis de Biomasa Lignocelulósica. (Tesis para optar por el grado de Doctorado en Ingeniería Química), Santa Fe, Argentina: Universidad Nacional del Litoral, 2016.

[21] P. Azadi, O. R. Inderwildi, R. Farnood y D. A. King, "Liquid Fuels, Hydrogen and Chemicals from Lignin: A Critical Review", Renewable and Sustainable Energy Reviews, vol. 21, pp. 506-523, 2013.

[22] J. Contreras, Efecto de la Tempereatura y la Concentración de Ácido Sulfúrico en el Perfil de Azúcares Obtenido del Pretratamiento de Aserrín de Pinus Oocarpa (Proyecto de graduación para optar por el grado de Licenciatura en Ingeniería Agroindustria Alimentaria), Zamorano, Honduras: Universidad Zamorano, 2001.

[23] D. Corredor, Pretreatment and Enzymatic Hydrolysis of Lignocellulosic Biomass. (Tesis para optar por el grado de Doctorado), Kansas, Estados Unidos: Kansas State University, 2008.

[24] B. P. Lavatack, G. J. Griffin y D. Rodman, "The Acid Hydrolysis of Sugarcane Bagasse Hemicellulose To Produce Xylose, Arabinose, Glucose and Other Products", Biomass and Bioenergy, vol. 23, $\mathrm{n}^{\circ}$ 367-380, 2002.

[25] C. Chandler, N. Villalobos, E. González, E. Arenas, Z. Mármol, J. Ríos y C. A. Mazzarri, "Hidrólisis ácida diluida en dos etapas de bagazo de caña de azúcar para la producción de azúcares fermentables", Multiciencias, vol. 12, n 3, pp. 245-253, 2012.

[26] N. Frederick, N. Zhang, A. Djioleu, X. Ge, J. Xu y D. J. Carrier, "The effect of washing dilute acid pretreated poplar biomass on ethanol yields.", de Sustainable degradation of lignocellulosic biomass - techniques, applications and commercialization, INTECH, 2013.

[27] Hach Company, Manual de análisis de agua, Colorado, EEUU, 2000.

[28] M. Córdoba, Determinación del Efecto de la Concentración de la Base $\mathrm{NaOH}$, de la Celulasa y Celobiasa en la Hidrólisis para la Producción de Etanol a Partir del Rastrojo de la Piña. (Proyecto de graduación para optar por el grado de Licenciatura en Ingeniería Química), San José, Costa Rica: Universidad de Costa Rica, 2011.

[29] B. Melissari, «Comportamiento de Cenizas y su Impacto en Sistemas de Combustión de Biomasa,» Memoria de trabajo de Difusión Científica y Técnica, vol. 10, pp. 69-82, 2012.

[30] M. Villegas, Evaluación del Efecto del Tamaño de Partícula, Concentración de Enzimas y Tiempo en la Hidrólisis y Fermentación Integradas para la Producción de Etanol. (Proyecto de graduación para 
optar por el grado de Licenciatura en Ingeniería Química)., San José, Costa Rica: Universidad de Costa Rica, 2010.

[31] Y. Fahmy, T. Mobarak, M. Sakhawy y M. Fadl, "Agricultural Residues (Wastes) for Manufacture of Paper, Board, and Miscellaneous Products: Background Overview and Future Prospects", International Journal of Chem Tech Research, vol. 10, nº 2, pp. 424-448, 2017.

[32] J. L. Martínez Altamiranda y D. Z. Fernández de Castro, Estudio Inicial de la producción de etanol a partir de las hojas de palma de aceite mediante un proceso de fermentación y sacarificación simultanea (SFS), Bogota, 2016.

[33] H. G. Chico León y D. A. Sandoval Rojas, Determinación de las concentraciones de enzimas Celulasa y B-Glucosidasa para la obtención de azúcares fermentables a partir de cascarilla de arroz, Nuevo Chimbote-Peru, 2015.

[34] R. Aguilar, J. A. Ramírez, G. Garrote y M. Vázquez, "Kinetic study of the acid hydrolysis of sugar cane bagasse", Journal of Food Engineering, vol. 55, nº 4, pp. 309-318, 2002.

[35] R. G. Candido, G. G. Godoy y A. R. Gonçalves, "Study of Sugarcane Bagasse Pretreatment with Sulfuric Acid as a Step of Cellulose Obtaining", International Journal of Biological, Biomolecular, Agricultural, Food and Biotechnological Engineering, vol. 6, nº 1, pp. 6-10, 2012.

[36] I. Soares, K. Mendes, M. Benachour y C. Abreu, "Evaluation of the Effects of Operational Parameters in the Pretreatment of Sugarcane Bagasse with Diluted Sulfuric Acid Using Analysis of Variance", Chemical Engineering Communications, vol. 204, nº 12, pp. 1369-1390, 2017.

[37] E. Ixcotoyac, Efecto de la Temperatura y Concentración de Ácido Sulfúrico de Pretratamiento de Aserrín de Pinus spp. en la Producción de Azúcares y 5-Hidroximetilfurfural. (Proyecto de graduación para optar por el grado de Licenciatura en Ingeniería en Agroindustria Al, Honduras: Universidad Zamorano, 2013.

[38] Y. Sun y J. Cheng, "Hydrolysis of Lignocellulosic Materials for Ethanol Production: a Review", Bioresource Technology, vol. 83, pp. 1-11, 2002.

[39] A. Redding, Z. Wang, D. Keshwani y J. J. Cheng, "High Temperature Dilute Acid Pretreatment of Coastal Bermuda Grass for Enzymatic Hydrolysis", Bioresourse Technology, vol. 102, n 2, pp. 14151424, 2011.

[40] H. Montaño, S. Rincón y J. Serrato, «Study of the Influence of Dilute Acid Pre-Treatment Conditions on Glucose Recovery from Moringa Oleifera Lam for Fuel-Ethanol Production.,» International Journal of Green Energy, vol. 14, nº 7, pp. 613-623, 2017.

[41] Y. H. Jung, I. J. Kim, H. K. Kim y K. H. Kim, "Dilute acid preteatment of lignocellulose for whole slurry ethanol fermentation”, Bioresourse Technology, vol. 132, pp. 109-114, 2013.

[42] S. Choojit, T. Ruengpeerrakul y C. Sangwichien, "Optimitation of acid hydrolysis of pineapple leaf residue and bioconverion to ethanol by saccharomyces Cerevisiae", Cellulose Chemistry and Technology, vol. 52, nº 3-4, pp. 247-257, 2018.

[43] C. Gámez, Efecto de la Temperatura y la Concentración de Ácido Sulfúrico en el Pretratamiento para la Producción de Bioetanol a partir de Estiércol de Ganado Lechero. (Proyecto especial para optar por el grado de Licenciatura en Ingeniería Agroindustria Alimentaria, Honduras: Universidad Zamorano, Francisco Morazán, 2008. 
[44] G. Chen y Z. Liu, "Enzymatic Hydrolysis of Lignocellulosic Biomass from Low to High Solids Loading”, Engineering in Life Sciences, vol. 17, n 5, pp. 489-499, 2016.

[45] A. Tanksale, J. N. Beltramini y G. Q. Lu, "Reaction Mechanisms for Renewable Hydrogen from Liquid Phase Reforming of Sugar Compounds", Developments in Chemical Engineering and Mineral Processing, vol. 14, nº 1-2, pp. 9-18, 2006.

[46] M. L. Barbelli, Reacciones en Fase Líquida para la Obtención de Hidrógeno y Biopropilenglicol a Partir de Glicerol. (Tesis para optar por el grado de Doctorado en Ingeniería)., La Plata, Argentina: Universidad Nacional de La Plata, 2016.

[47] I. Coronado, M. Stekrova, L. García, M. Reinikainen, P. Simell, R. Karinen y J. Lehtonen, “Aqueous-phase Reforming of Methanol Over Nickel-based Catalysts for Hydrogen Production", Biomass and Bioenergy, vol. 106, pp. 29-37, 2017.

[48] G. Wen, Y. Xu, Z. Xu y Z. Tian, "Characterization and Catalytic Properties of the Ni/Al2O3 Catalysts for Aqueous-phase Reforming of Glucose", Catalysis Letters, vol. 129, n 1-2, pp. 250-257, $2008 \mathrm{a}$.

[49] G. Pipitone, A. Frattini, S. Bocchini, R. Pirone y R. Bensaid, "Aqueous Phase Reforming of Sugar-Based Biorefinery Streams: from the Simplicity of Model Compounds to the Complexity of Real Feeds", Catalysis Today, vol. 345, pp. 267-279, 2019.

[50] G. Wen, Y. Xu, H. Ma, Z. Xu y Z. Tian, "Production of Hydrogen by Aqueous-Phase Reforming of Glycerol”, International Journal of Hydrogen Energy, vol. 33, nº 22, pp. 6657-6666, 2008b.

[51] B. Meryemoglu, B. Kaya, S. Irmak, A. Hesenov y O. Erbatur, " Of Batch Aqueous-Phase Reforming of Glycerol and Lignocellulosic Biomass Hydrolysate”, Fuel, vol. 97, pp. 241-244, 2012. 